\title{
Pharmacological Activities and Progress in Structure Modification of Salidroside
}

\author{
Xuewei Luo, Na Bao, Li Chen* and Jianbo Sun
}

Department of Natural Medicinal Chemistry, China Pharmaceutical University, 24 Tongjia Lane, Nanjing, 210009, China.

\begin{abstract}
Salidroside is a phenylpropanoid glycoside isolated from Rhodiola rosea $L$., a traditional Chinese medicinal (TCM) plant, and has displayed a broad spectrum of pharmacological properties, such as resisting anoxia, antiradiation and antifatigue. In addition, it has low toxicity as traditional Chinese medicinal plant. However, the bioavailability of salidroside is low because of its instability for hydrolase. Lots of researchers modified the structure of salidroside to enhance its bioactivity. This paper summarizes the structure modification and activities study of salidroside in recent ten years, and preliminary discuss the structure-activity relationship of salidroside analogues, which hope to provide new ideas of modification in future.
\end{abstract}

Keywords: Salidroside; Analogues: Anti-radiation; Anti-tumor; Resisting anoxia

\section{Introduction}

Salidroside is the active ingredient isolated from tuber and root tuber of Rhodiola rosea L., a traditional Chinese medicinal (TCM) plant. In Shennong Classic of Materia Medica, the ancient people regarded R. rosea as the best supplement which can tonify the kidney, promote blood circulation to stop bleeding and clear lung to stop coughing. The Compendium of Materia Medica recorded R. rosea as the rare tonic and had been used for the treatment of chest congestion, nausea, weakness, etc. Modern pharmacological studies have demonstrated that salidroside from R. rosea is the main bioactive compound with many biological activities, such as resisting anoxia, anti-radiation, antifatigue, improving oxygen lack, anti-tumor, preventing cardiovascular and postponing aging [1].<smiles>OC[C@H]1O[C](COCCc2ccc(O)cc2)[C@@H](O)[C@H](O)C1O</smiles>

Salidroside

\section{Pharmacological activities}

Anti-tumor effect: Salidroside has been proved to have antitumor effect with inhibiting the growth of various tumor cells, such as hepatoma QGY-7703, hepatoma SMMC-7721, hela cells, human gastric adenocarcinoma SGC-7901 cells and SW1116 cells [2-6], etc. Many studies showed that salidroside is possible to block cancer cancer cells growth [7], inhibit migration and invasion of cancer cells [6], significantly decrease neovascular reaction [8], induce cancer cells differentiate to normal cells [9] and has immunity efficacy for tumorbearing mice $[10,11]$.

Due to obvious inhibitory activities of salidroside on many kinds of tumor cells, the mechanisms of salidroside were summarized according to the corresponding tumor cell lines.

Hepatoma H22:He [12] had shown that salidroside can inhibit hepatoma $\mathrm{H} 22$ solid tumor growth across dose-time-effect dependencies. The inhibitory rates of salidroside for $\mathrm{H} 22$ solid tumor in low, medium, high doses (20, 40, $80 \mathrm{mg} / \mathrm{kg}$ ) were $28.9 \%, 43.3 \%, 53.5 \%$.
After being compatible with cyclophosphamide (CTX) at small dose $(10 \mathrm{mg} / \mathrm{kg})$, the inhibitory rates of salidroside for H22 solid tumor in different doses were $35.2 \%, 48.0 \%, 55.9 \%$, which were better than the inhibitory rates of CTX (34.1\%). In addition, the spleen and thymus weights of each dose group were obviously increased. This suggests that salidroside has strong anti-hepatoma effect in vivo and in vitro, and when it has been compatible with CTX, it can increase the antihepatoma effect of CTX with decreasing toxicity.

Study on the related mechanism: 1. Salidroside has immunopotentiation effect (improving the mice spleen index and thymus index; improving the activation capacity for $\mathrm{T}$ lymphocytes and NK cells; improving secretion level of IL-2, IL-12 in tumor-bearing mice's serum [13]). 2. Salidroside can inhibit DNA Topo II activity and present a certain dose-effect relationship [12].

SW1116 cells: Sun found that high concentrations of salidroside $(10,20,50 \mu \mathrm{g} / \mathrm{ml})$ can significantly inhibited proliferation of SW1116 cells in a parallelly [16]. Through western blot they found that salidroside decreased proteins expression of phosphorylation levels in JAK2/STAT3 signaling, while MMP-2 and MMP-9 proteins levels were decreased and protein expression of VEGF and VEGFR-2 were downregulated.

Human Skin Melanoma A375 cells: Yang found that saldroside can inhibit invasion and metastasis of human skin melanoma A375 cells with the dose-effect dependence [13]. The inhibition rate reached $74.2 \%$ when the concentration of salisroside is $100 \mu \mathrm{g} / \mathrm{ml}$. This suggested that the mechanism may be related to salidroside's inhibiting adhesion for tumor cells to the base membrane.

*Corresponding authors: $\mathrm{H}$ Li Chen, Department of Natural Medicinal Chemistry, China Pharmaceutical University, 24 Tongjia lane, Nanjing 210009, China; Tel: 8625-83271415; E-mail: chenliduo@sohu.com

Jianbo Sun, Department of Natural Medicinal Chemistry, China Pharmaceutical University, 24 Tongjia lane, Nanjing 210009, China; Tel/Fax: 862583271415; Email: sjbcpu@gmail.com

Received March 04, 2017; Accepted March 15, 2017; Published March 21, 2017

Citation: Luo X, Bao N, Chen L, Sun J (2017) Pharmacological Activities and Progress in Structure Modification of Salidroside. Med Chem (Los Angeles) 7: 818-823. doi: 10.4172/2161-0444.1000434

Copyright: (c) 2017 Luo X, et al. This is an open-access article distributed under the terms of the Creative Commons Attribution License, which permits unrestricted use, distribution, and reproduction in any medium, provided the original author and source are credited. 
To sum up, salidroside can inhibit the growth of various tumor cells. When it is compatible with the chemotherapy drugs, it can increase their anti-tumor effects and decrease toxicities. Such characteristics mainly associated with salidroside's good improvement of the immune system function.

\section{Antioxidant activity}

Promote the biosynthesis of mitochondria: Mitochondria is the main place to produce ROS. When excessive ROS produced, mitochondria exposed to high concentration active oxygen environment which led to oxidative damage and affected the normal physiological function of cells. Therefore, promoting the biosynthesis of mitochondrial function and increasing the activity of antioxidant enzymes in mitochondria can decrease levels of ROS and reduce the oxidative damage of mitochondria [14].

Salidroside can promote the biosynthesis of mitochondria and improve numbers of mitochondria by activating the signal pathway controlled by PGC-1 1 through raising AMPK phosphorylation $[15,16]$.

Scavenge free radicals in cells: Free radicals, including superoxide anion, hydroxyl radical, NO free radical, alcoxyl radical and lipid peroxide (LPO), are widely-existing in human body. The oxygen free radical can induce starting the free radical chain reaction with its unstable property, and can continuously generate new active free radicals.

Investigation shows that salidroside can increase the activities of SOD (superoxide dismutase), GSH-Px (glutathione peroxidase), GSH (glutathione), and reduce the content of MDA (malondialdehyde) at the same time, which can speed scavenging free radicals $[17,18]$.

\section{Anti-Aging Effect}

When numbers of ROS exceeded in mitochondria, it would cause the DNA oxidative damage. Due to the lacking of integrated DNA damage repair system in the mitochondria, it causes oxidative damage accumulation, and gradually leads to mitochondrial dysfunction. Then it causes changes of physiological function and leads to the age-related diseases [19].

One of the main causes for aging is the mitochondrial dysfunction, salidroside can inhibit cell oxidative damage, maintain the numbers and function of mitochondria. Researches showed that salidroside could reduce the content of MDA and increase the activity of SOD and GSH-Px in the natural aging mice [20].

In addition, salidroside can enhance sports abilities and extend the memory time of aging mice induced by D-galactose. It also shows that salidroside can reverse aging by promoting mitosis of lymphatic cells and synthesis of IL-2 [21].

Anti-myocardial ischemia and hypoxia activities: Salidroside is an oxygen glycoside molecule with the basic pharmacophore to treat coronary atherosclerotic heart disease, and the activity is affected by the appropriate length of the connection chain between glycosyl and the polysubstituted aromatic ring.

Studies have shown the mechanisms of salidroside's antimyocardial ischemia and hypoxia activities [22]: 1. Reduce the synthesis of angiotensin converting enzyme, and obviously inhibit the proliferation and contraction of vascular smooth muscle cells. 2. Reduce the myocardial oxygen consumption, and strengthen the pumping force of heart to improve the symptoms of heart failure. 3 . Improve the activity of $\mathrm{Na}^{+}-\mathrm{K}^{+}$-ATP enzyme in cells, and reduce the content of sodium, calcium ions. 4. Scavenge oxygen free radicals and inhibit lipid peroxidation.

Hepatoprotective effect: Salidroside can attenuate the induced acute increase in serum aspartate aminotransferase and alanine aminotransferase activities, and lessen the levels of tumour necrosis factoralpha and serum nitric oxide. Wu found that salidroside could reduce the appearance of necrotic regions and expression of caspase-3 and hypoxia-inducible factor-1a in liver tissue. And salidroside perhaps worked as a precursor of GSH and free-radical scavenger to reduce cellular GSH depletion and balance ROS to ameliorate peroxide and superoxide activities [23].

Nerve cell protection: $A \beta$ ( $\beta$-amyloid) stimulates the activation of microglia cells, NADPH (nicotinamide adenine dinucleotide phosphate), iNOS (inducible Nitric Oxide Synthase), NF- $\kappa B$, and releases the TNF, an inflammatory factor which can directly or indirectly cause nervous system damage and nerve cells dysfunction.

Zhang used $\mathrm{AD}$ mice models induced by $\mathrm{A} \beta 1-40$ to examine the intervention effect for AD of salidroside [24]. The study found that salidroside may inhibit the NF- $\kappa B-i N O S-N O$ pathway. Salidroside can inhibit the expression of NF- $\kappa \mathrm{B}$ and reduce the expression of iNOS to suppress the generation of NO, which can finally alleviate neuron cell damage induced by oxidative stress. At the same time, salidroside can significantly reduce the amount of NO in cells and reduce excessive expression of iNOS and nNOS, thus it can inhibit the NO pathway and protect nerve cells.

Others: Salidroside can also inhibit inflammatory reaction, relieve cerebral edema and protect brain tissue [25-27].

The structural modification for salidroside and biological activities

Salidroside have broad application prospects and high medicinal values. However, the basic structure of salidroside is the oxygen glycoside, which is easily hydrolyzed by enzyme in vivo. This makes the bioavailability of salidroside low and cannot reach the effective blood drug concentration. In addition, on the base of pharmacokinetic research [28], salidroside only distributes over liver, kidney and heart which have the adequate blood supply. Due to its low fat soluble, salidroside cannot pass the blood brain barrier.

In order to enhance the bioavailability and bioactivity of salidroside, a lot of researchers have modified its structure. The structural modification is mainly focused on two aspects: the structural modification of tyrosol and glucoside.

\section{The Modification of Tyrosol}

Tyrosol is the aglycone of salidroside, which has been found to have antioxidant effects and could resist anoxia. Therefore, Zhu [29] considered adding the structure of chalcone to tyrosol, hoping to enhance the bioactivity of tyrosol, and finally got a series of novel compounds (Figure 1). The anti-radiation and anti-anoxia effects of novel compounds were better than tyrosol, and compound 1 exhibited the best activities, followed by compound 2 (tyrosl, DPPH $\mathrm{IC}_{50}=38.9$ $\mu \mathrm{g} / \mathrm{ml}$, FRAP $=12.58 \mathrm{mmol} / \mathrm{g}$; compound $1 \mathrm{DPPH} \quad \mathrm{IC}_{50}=13.20 \mu \mathrm{g} /$ $\mathrm{ml}$, FRAP $=18.25 \mathrm{mmol} / \mathrm{g}$; compound $2 \mathrm{DPPH} \quad \mathrm{IC}_{50}=17.60 \mu \mathrm{g} / \mathrm{ml}$, FRAP $=16.15 \mathrm{mmol} / \mathrm{g}$ ).

Comparing the $\mathrm{IC}_{50}$ values of FRAP, DPPH and structures of the synthesized compounds, we found that the adjacent hydroxyl on benzene ring (Figure 2) can enhance anti-radiation activity. It enhances the stability of oxygen free radicals through hydrogen bonding interaction. 


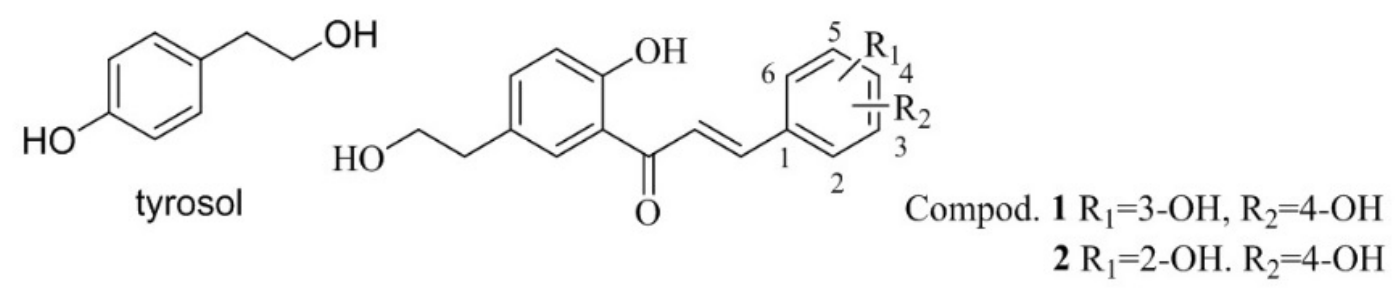

Figure 1: Stuctures of tyrosol and some related modification compounds

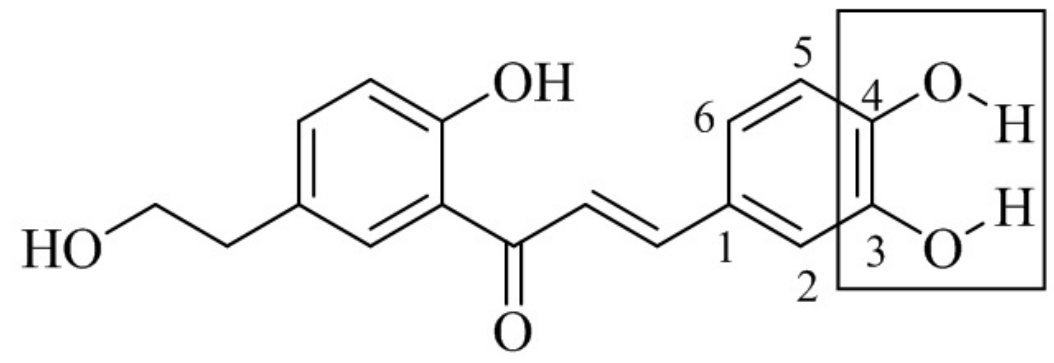

Figure 2: The adjacent hydroxyl on benzene ring in modified compounds.

The experimental results show that increasing the numbers of hydroxyl on benzene ring can increase activities of anti-radiation and anti-anoxia. However, the number of hydrogen bond donor (hydroxyl, amino, etc.) must be less than five according to "drugs 5 rule".

\section{The Modification of Glucoside}

Most researchers usually choose four main points to modify the glucoside of salidroside: 1 . The groups on the benzene ring; 2 . The length and saturability of carbon chain between benzene ring and glycosyl donor; 3 . The type of glycosidic bond; 4 . The type and configuration of glycosyl donor. Most modification focused not only on a single site, but usually on multiple sites.

Chen modified the structure of salidroside from five aspects [30]: 1. Change phenol hydroxyl groups into electron-donating groups or electron-withdrawing groups. 2. Change the position of the phenol hydroxyl groups. 3. Add electron-donating group or electronwithdrawing group into o-position or p-position of phenol hydroxyl groups. 4. Change numbers of carbons for link chain from two to one. 5. Change the glycosidic bond. Finally the researcher got 47 novel compounds and tested their abilities of anti-anoxia under normal pressure, $\mathrm{OD}$ value and $\mathrm{DPPH}^{\mathrm{E}} \mathrm{C}_{50}$.

In the test of anti-anoxia activity under normal pressure, compounds 3 and 4 (Figure 3) had the quite activities in different doses $(16.6,50$, $150 \mathrm{mg} / \mathrm{kg} /$ day) compared with salidroside (salidroside, $50 \mathrm{mg} / \mathrm{kg}$ / day, the survival time $42.8 \pm 5.1 \mathrm{~min}$; compound $3,50 \mathrm{mg} / \mathrm{kg} / \mathrm{day}$, the survival time $40.1 \pm 6.4 \mathrm{~min}$; compound $4,50 \mathrm{mg} / \mathrm{kg} /$ day, the survival time $44.1 \pm 6.0 \mathrm{~min}$ ). It is speculated that $\mathrm{S}$-glycosides and C-glycosides have better stability to enhance anti-anoxia activity.

In the test of OD value in endotheliocyte, the activity of compound 5 (Figure 4) was better than salidorside (OD values of model, salidroside and compound 5 were $0.301 \pm 0.018,0.473 \pm 0.010$ and $0.533 \pm 0.012$, respectively). It could speculate that the introduction of methylenedioxy can enhance the stability for acid, alkali and enzyme. Methylenedioxy is the isosteres for oxygen, which not only can largely change activities of compounds but also can enhance the stability for acid, alkali and enzyme [31,32]. In addition, the introduction of amine structures can improve $\log \mathrm{P}$ of compounds, thus it can enhance the stability and bioavailability.

In the test of DPPH $\mathrm{EC}_{50}$, the activities of compounds $\mathbf{6}, 7,8$ and 9 (Figure 5) were stronger than salidroside and Vc (Vc DPPH $\mathrm{EC}_{50}=14.5$; salidroside $\mathrm{DPPH} \mathrm{EC}_{50}=17.1$; compound $6 \mathrm{DPPH} \mathrm{EC} \mathrm{E}_{50}=$ 12.5; compound $7 \mathrm{DPPH} \mathrm{EC} \mathrm{E}_{50}=10.4$; compound $8 \mathrm{DPPH} \mathrm{EC}_{50}=10.0$; compound $9 \mathrm{DPPH} \mathrm{EC}_{50}=11.0$ ). It is speculated that the introduction of nitro on the benzene ring can enhance the ability of scavenging free radicals. When the nitro is at 2 or 3 site, the ability of scavenging free radicals is better than others. In addition, the ability of scavenging free radicals for S-glycosides and $\mathrm{N}$-glycosides are better than $\mathrm{O}$-glycosides.

To sum up, the properties and positions of substituent on the benzene ring, the type of glycosidic bond and glycosyl can influence the activity ofsalidroside. The introduction of methylenedioxy, $\mathrm{N}$-glycoside, S-glycoside and C-glycosides can increase the stability and pharmacological activities.

Peng designed and synthesised a series of C-glycosides and tested myocardial protection [33].

The main modification sites were: 1 . Change phenol hydroxyl groups into electron-donating groups or electron-withdrawing groups. 2. Introduce amine structures on the connection chain. 3. Change $\mathrm{O}$-glycoside into C-glycoside.

In the test of $\mathrm{LDH}$ inhibition rate, compounds 10, 11, 12, 13 and $\mathbf{1 4}$ in different doses were better than salidroside with obvious concentration-response relationship (Table 1).

Compound $\mathbf{1 1}$ is the best in different dosage group. It is speculated that the introduction of halogen atoms or methoxyl to $o-, m-, p$ - position on the benzene ring can improve myocardial protection, and the introduction of fluorine atoms had the best protection. C-glycosides. had stronger protection than O-glycosides.

Zhang [34] modified structure of salidroside from following 
<smiles>COc1cc(CSC2OC(O)C3(O)COC2C(O)C3O)cc(OC)c1OC</smiles>

3<smiles>OCC1(CO)OC(CNCc2ccccc2Cl)C(O)C(O)C1O</smiles>

4

Figure 3: Stuctures of compounds 3 and 4.

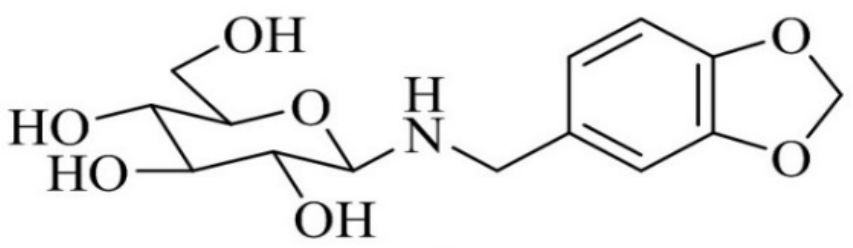

5

Figure 4: Stucture of compound 5.

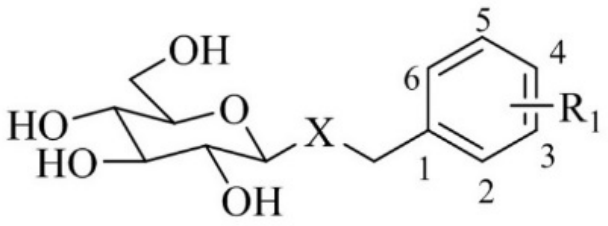
Compod. $6 \mathrm{X}=\mathrm{S} ; \quad \mathrm{R}_{1}=3-\mathrm{NO}_{2}$
$7 \mathrm{X}=\mathrm{NH} ; \mathrm{R}_{1}=2-\mathrm{NO}_{2}$
$8 \mathrm{X}=\mathrm{NH} ; \mathrm{R}_{1}=2-\mathrm{NO}_{2}$
$9 \mathrm{X}=\mathrm{NH} ; \mathrm{R}_{1}=4-\mathrm{NO}_{2}$

Figure 5: Stuctures of compounds 6, 7, 8 and 9

\begin{tabular}{|c|c|c|c|}
\hline \multirow{2}{*}{ Compound } & \multicolumn{3}{|c|}{ LDH inhibition rate } \\
\cline { 2 - 4 } & $\mathbf{1 0 0 0} \mathbf{~ g g / m l}$ & $\mathbf{2 0 0} \mathbf{\mu g} / \mathbf{m l}$ & $\mathbf{4 0} \mathbf{~ g} / \mathbf{m l}$ \\
\hline Salidroside & $22.77 \pm 12.00$ & $30.74 \pm 14.30$ & $48.10 \pm 6.88$ \\
\hline 10 & $29.67 \pm 19.40$ & $42.02 \pm 16.55$ & $82.85 \pm 24.25$ \\
\hline 11 & $67.59 \pm 45.83$ & $82.43 \pm 43.35$ & $92.56 \pm 42.12$ \\
\hline 12 & $36.62 \pm 27.65$ & $56.77 \pm 56.14$ & $73.89 \pm 35.51$ \\
\hline 13 & $30.99 \pm 20.45$ & $61.16 \pm 42.08$ & $66.53 \pm 47.34$ \\
\hline 14 & $26.18 \pm 27.26$ & $38.00 \pm 26.91$ & $49.83 \pm 25.37$ \\
\hline
\end{tabular}

Table 1: The LDH inhibition rate of Salidroside analogues in different doses.

aspects: 1. Remove phenolic hydroxyl groups; 2. Change carbon numbers of connection chain; 3. Introduce alkenes structure to connection chain; 4 . Change glycosyl into galactose. They got a series of novel compounds and test anti-fatigue activity. The anti-fatigue activities of novel compounds were weaker than salidroside. The result showed that phenolic hydroxy is the active site for anti-fatigue activity. The anti-fatigue activities of galactose groups were better than glucose groups, which showed that galactose can enhance anti-fatigue activity. The three-dimensional structures of carbohydrate are different, and biological activities of polysaccharides are determined by glycosyls. So different types of glycosyl have an influence on biological activities of salidroside.

Many studies have shown that compounds with glucosamine have nerve cells protection [35]. Guo changed glycosyl donors into $\mathrm{N}$-acetylglucosamine, glucosamine and synthesized compounds 15,16
(Figure 6) [36]. They studied the mechanism of protecting PC12 nerve cells.

Pretreatment with compounds 15, 16 and salidroside at different concentrations $(80,160,320 \mathrm{mg} / \mathrm{ml})$ significantly attenuated the cell viability loss evoked by hypoglycemia and serum limitation, and the attenuating effect on the survival of cultured PC12 cells displayed a dose dependent pattern. The changes in the percentage of apoptotic cells were also analyzed by Hoechst 33342 staining. In comparison to control group, exposure to hypoglycemia and serum limitation alone produced significantly apoptosis. Pre-incubation with 80,160 , and $320 \mathrm{mg} / \mathrm{ml}$ of compounds $\mathbf{1 5}, 16$ and salidroside, the apoptotic percentages were significantly lower than that produced by exposure to hypoglycemia and serum limitation alone. Western blot analysis showed that compounds 15 and 16 against hypoglycemia and serum limitationinduced cell apoptosis in the PC12 cells partly attributed to their evoked modulation of apoptosis-related gene expression(especially Bcl-2), which was in accordance with salidroside. Therefore the introduction of $\mathrm{N}$-acetylglucosamine can enhance the protection of PC12 cells by inhibiting apoptosis gene (Bcl-2) expression, inhibiting cell apoptosis and enhancing cell viability.

Zheng modified structure of salidroside from following aspects [37]:

1. Introduce more phenolic hydroxyl or methoxyl groups on the benzene ring 


\section{Change the carbon numbers of connection chain}

3. Change the configuration of glycosyl. They finally got 32 novel compounds and tested anti-anoxia ability. Compounds 17, 18, 19, 20 (Figure 7) were better than salidroside and $\mathrm{Vc}\left(\mathrm{Vc} \mathrm{EC}_{50} / \mu \mathrm{M}=54.61\right.$; salidroside $\mathrm{EC}_{50} / \mu \mathrm{M}>100$; compound $17 \mathrm{EC}_{50} / \mu \mathrm{M}=35.85$; compound $18 \mathrm{EC}_{50} / \mu \mathrm{M}=36.71$; compound $19 \mathrm{EC}_{50} / \mu \mathrm{M}=38.05$; compound $20 \mathrm{EC}_{50} /$ $\mu \mathrm{M}=39.73$ ).

Compound 17 have exhibited the best anti-anoxia activity. Structure and activity relationships (SARs) revealed that: 1 . Introduction of more phenol hydroxyl groups on the benzene ring can improve antioxidant activity; 2 . The potency of $\beta$-glucoside is better than $\alpha$-glucoside; 3 . when containing two carbons in linker manifested best activity.

Compound $\mathbf{1 7}$ and $\mathbf{1 8}$ then were investigated for their protective effect on human normal liver cells and the mechanism. Noticeably, compounds 17 and 18 comparing with salidroside showed better cell protection ( $\mathrm{CCl}_{4}$ mediated apoptosis). And salidroside showed good cell protection merely in the concentration for $100 \mathrm{mM}$ compounds $18(50,100 \mathrm{mM})$ and compounds $17(10,50,100 \mathrm{mM})$ in low doses showed better cell protective effect.

In an effort to detect the activities of SOD, GSH-Px and the content of MDA in human liver apoptotic cells, we further decided to add 10 $\mathrm{mM} \mathrm{CCl}_{4}$ into LO2 cells, we found the activity of GSH-Px was reduced by $51 \%$, SOD was reduced by $53 \%$, and content of MDA was raised by $70 \%$. Through treated with compounds 17,18 and salidroside, the activities of GSH-Px and SOD activities in LO2 cells were enhanced. The toxicity caused by $\mathrm{CCl}_{4}$ was accompanied by increased lipid peroxides. And the activities of compounds 17, 18 were better than salidroside (salidroside $100 \mathrm{mM}$, SOD 61\%, GSH-Px 61\%, MDA 157\%; compound 17100 mM, SOD 89\%, GSH-Px 86\%, MDA 110\%; compound 18100 $\mathrm{mM}$, SOD $83 \%$, GSH-Px $80 \%$, MDA $117 \%)$. It can be speculated that compounds 17 and 18 can inhibit the production of peroxide, scavenge free radicals, and inhibit apoptosis mediated by $\mathrm{CCl}_{4}$

Preliminary discussion of structure-activity relationship for salidroside analogues

To sum up, aglycone, chain between aglycone and glycosyl, the type of glucosidic bond and the configuration of glycosyl can affect the biological activities of salidroside analogues.

The preliminary structure-activity relationship studies can be concluded as follows:

1. Substituents on the benzene ring of aglycone. Phenolic hydroxy is the active site for anti-fatigue and anti-oxidant activities [34]. When no phenol hydroxyl was introduced, the antioxidant activity could be reduced. And when the length of phenolic hydroxyl increases, the antioxidant activity improved [29, 37]; The positions of phenol hydroxyl also had the had certain differences to antioxidant activity. When phenol hydroxyl is at position $\mathrm{C} 1$, the antioxidant activity has no influence. When phenol hydroxyl is at position C2 or C6, the antioxidant activity is reduced. When phenol hydroxyl is replaced by the electron withdrawing group, the hydrogen on the benzyl carbon is tend to leave, which can enhance the ability of catching DPPH and show better antioxidant activity[30]; When introduces electron-donating groups to position $\mathrm{C}_{2}$ or $\mathrm{C}_{6}$ on the benzene ring, the antioxidant ability is enhanced. It also can improve the protection for myocardial cells [33]; whereas replacing methoxyl to position $\mathrm{C}_{2}$ or $\mathrm{C}_{6}$, the antioxidant activity is reduced [30].

2. The influence of the link chain. Various length of chain have differences to antioxidant activity, when the carbon is only one, the anti-hypoxia activity is shinking and antioxidant activity is reduced $[30,37]$.

3. The type of glucosidic bond. S-glycosides, C-glycosides and $\mathrm{N}$-glycosides exbited better antioxidant activity than O-glycosides. It can be speculated that $\mathrm{S}$-glycosides, C-glycosides and $\mathrm{N}$-glycosides have better stability in vivo [30]. And $\mathrm{N}$-glycosides have better water-soluble. It can enhance the combination of compounds with receptors; When there exits amine groups on benzene ring, the electron cloud on the benzyl carbon redistributes due to strong electron-withdrawing effect. It tends to form more stable $\mathrm{C}-\mathrm{N}$ double bond with amino, which lead to increased numbers of free oxygen. However, oxygen atoms cannot combine with carbon atoms to form a more stable structure, so the number of free oxygen is less. Therefore the ability of scavenging free radicals of $\mathrm{N}$-glycosides is better than $\mathrm{O}$-glycosides [30, 33].

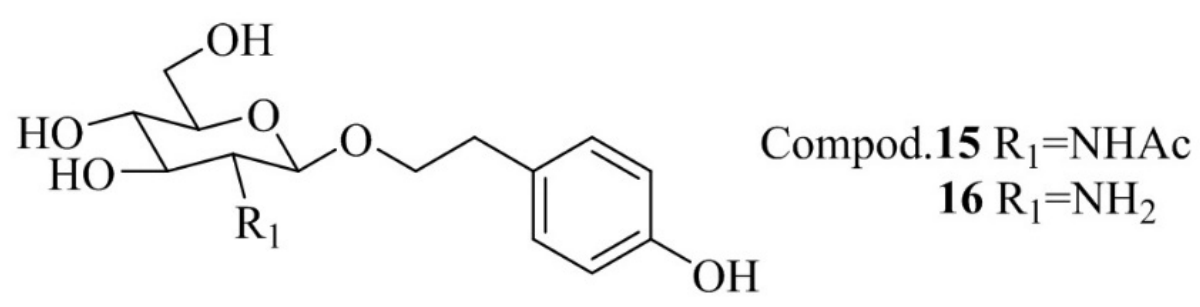

Figure 6: Stuctures of compounds 15 and 16

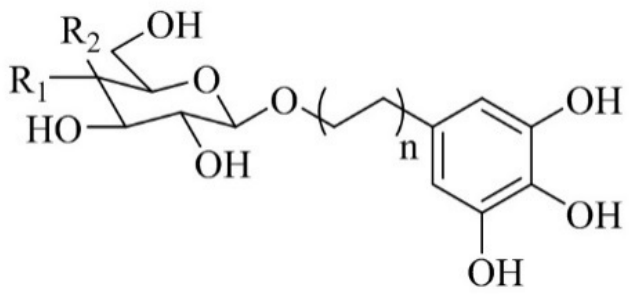

Compod. $17 \mathrm{n}=2 ; \mathrm{R}_{1}=\mathrm{H} ; \mathrm{R}_{2}=\mathrm{OH}$

$18 \mathrm{n}=2 ; \mathrm{R}_{1}=\mathrm{PH} ; \mathrm{R}_{2}=\mathrm{H}$

$19 \mathrm{n}=3 ; \mathrm{R}_{1}=\mathrm{H} ; \mathrm{R}_{2}=\mathrm{OH}$

$20 \mathrm{n}=3 ; \mathrm{R}_{1}=\mathrm{OH} ; \mathrm{R}_{2}=\mathrm{H}$

Figure 7: Structures of compounds 17, 18, 19 and 20. 
4. The type and configuration of glycosyl. The anti-fatigue activities of galactose groups were better than glucose groups [34], and the introduction of $\mathrm{N}$-acetylglucosamine can enhance the protection of PC12 cells [36]. The antioxidant activity of $\beta$-glucoside is better than a-glucoside.

\section{Conclusion}

Salidroside is a phenylpropanoid glycoside isolated from Rhodiola L. which which have been extensively used for the treatment of resisting anoxia, anti-radiation and antifatigue in Traditional Chinese Medicines. In addition, it has anti-tumor, radioresistance, antiviral and cytoprotection effects. Due to its low toxicity, salidroside has been promising used in clinic. However, drugs about salidroside on the market at present mainly tend to cosmetics. The main reason is that the structure of salidroside is $\mathrm{O}$-glycosides, which have low metabolism stability. This paper review on the structural modifications and activities study of salidroside in recent ten years, and the preliminary structureactivity-relationship of salidroside analogues, which intend to provide perspectives for rational modification of salidroside for antioxidant and acetylcholinesterase inhibitory activities, along with the other potential pharmacological activities.

\section{Conflict of interest}

The authors confirm that this article content has no conflict of interest.

\section{References}

1. Han XJ, Guo N, Zhu, MX, Yu T (2015) Research progress in pharmacological activities and related mechanism of salidroside. Chin J Biological Pharms 35: $171-175$

2. Yang J, Liu YL (2001) Dissection of key events in tubular epithelical to myfibroblast transition and its implication in renal interstitial fibrosis. Am J Pathol 159: 1465-1475

3. Razzzque MS, Taguchi T (2002) Cellular and molecular events leading to renal tubulointerstitial fibrosis. J Med Electron Microsc 35: 68-80.

4. Xu YQ, Pan L, Wang YH, Wang XT, Wang Z, et al. (2009) Effect of Safflower on tubular epithelial-myofibroblast transdifferentiation in renal interstitial fibrosis rats. Chin J Gerontol 29: 1344-1346.

5. Wei LL, Mo SR (2012) Inhibitory Effect of Rhodiola on Proliferation in Human Gastric Carcinoma Cells SGC-7901 and the Preliminary Study of its Anti-tumor Effect. Guangxi Med Univ.

6. Sun KW, Xia HW, Xia RL (2015) Anticancer effect of salidroside on colon cancer through inhibiting JAK2/STAT3 signaling pathway. IntJClinExpPathol 8: 615-621.

7. Wei LL, Mo SR (2015) An in vitro study of the anticancer effect of salidroside on human gastric carcinoma SGC-7901 cells J Shandong. Univ Health Sci 53: 12-15.

8. Skopinska-Rozewska E, Malinowski M, Wasiutynski A, Sommer E, Furmanowa $\mathrm{M}$, et al. (2008) The influence of Rhodiola quadrifida $50 \%$ hydro-alcoholic extract and salidroside on tumor-induced angiogenesis in mice. Pol $\mathrm{J}$ Vet Sci 11: $97-104$.

9. Xie FW, OuYang XN, Jiang MD (2006) Reverse effects of salidroside on expression of c-myc in SMMC-7721 cell line in vitro. Med J Natl Defending Forces Southwest China 16: 130-140.

10. Zhao W, Jiang DS, Bian QR, Ma XT, Wang TX, et al. (2000) Effects of Rhodiola rosea on Immune Function and its Anti-tumor Action in mice. J Acta Nutrimenta Sinica 22: 90-91.

11. Song HJ, Lv SC, Li LJ, Liu DP, Yin XZ, et al. (2011) The anti-tumor function of the salidroside. Chin J Gerontol 31: 3991-3992.

12. He XX, Mo SR (2014) Anti-hepatocarcinoma effect of salidroside and its mechanism [D]. Guangxi Med Univ.

13. Yang AR (2012) The effect of salidroside on the invasion ability of melanoma cells A375 in human skin. J Qinghai Med Coll 33: 56-58.
14. Fu YL (2010) Oxidative stress and Alzheimer's disease and the reviewe of oxidation treatment. J Foreign Med Sci: Psychiatry 37: 336-340.

15. Shen JJ, Wang Z (2013) Effects of salidroside on mitochondrial biogenesis and screening for anti-cancer compounds [D]. Peking. Uni Med Coll.

16. Wang $\mathrm{H}$ (2013) Adiponectin attenuates hypoxia/reoxygenation injury in neonata rat cardiomyocytes through improving PGC-1a-mediated mitochondrial biogenesis [D]. Fourth Mil Med Univ.

17. Wang JY, Yu R (2009) Protective Effect and Its Mechanism of Salidroside against Cerebral Ischemia-Reperfsion Injury in Rats [D]. Fudan Univ.

18. Wang JY, Yu R, Yao MH (2008) Protective Effect and Its Mechanism of Salidroside against Cerebral Ischemia-Reperfsion Injury in Rats. Chin J Tradit Chin Med Pharm.

19. Dai DF, Rabinovitch PS, Ungvari Z (2012) Mitochondria and cardiovascular aging. Circ Res 110: 1109-1124.

20. Shen JJ, Yuan LG, Li DD, Wang Z (2012) Research on the anti-aging role of salidroside in naturally aged mice. Chin Med Biotechnol 7: 412-417.

21. Mao GX (2010) Protective role of salidroside against aging in a mouse mode induced by D-galactose. Biomed Environ Sci 23: 161-166.

22. Li YL, Su QJ, Li LZ (2007) Advances on the pharmacological activities of salidroside[J]. Acta Academiae Medicinae 16: 98-100.

23. Wu YL, Lian LH, Jiang YZ, Nan JX (2009) Hepatoprotective effects of salidroside on fulminant hepatic failure induced by D-galactosamine and lipopolysaccharide in mice. J Phar Pharma 61: 1375-1382.

24. Zhang J (2012) Effect and mechanism of salidroside on cognitive deficits of Alzheimer's disease model rats induced by $\beta$-amyloid[D]. Hebei Med Univ.

25. Cao LL, Du GH, Wang MW (2005) Effect of salidroside on mitochondria injury induced by sodium azid. Acta Pharmaceutica Sinic 40: 700-704.

26. Gao J, Zhou R, You XT, Luo F, He H, (2016) Salidroside suppresses inflammation in a D-galactose-induced rat model of Alzheimer's disease via SIRT1/NF-KB pathway[J]. Metab Brain Dis 31: 771-778.

27. Peng S, Song SZ, Jiang S, Li X, Yao YL, Wu YL, et al. (2016) Salidroside Regulates Inflammatory Response in Raw 2647 Macrophages via TLR4/TAK1 and Ameliorates Inflammation in Alcohol Binge Drinking-Induced Liver Injury[J] Molecules 21: 1490.

28. Lin XF, Yu HX, Tan C, Zhang L, Chen B, et al. (2006) Radioiodine-labeling of salidroside and its biodistribution in mice. Nuclear Tech Sci 29: 913-916.

29. Zhu H, Luo ZL, Gu JW, Yan T, Du JH, et al. (2013) Design and Synthesis of Salidroside Derivates as Novel Radical Scavenging Antioxidants. Pharm J Chin PLA 29: 290-293.

30. Chen H (2012) Synthesis of Salidroside analogues with a carbon reduce on side chain and preliminary study of their anti-hypoxic and antioxidant activities [D]. Tianjin Med Univ.

31. Martin-pastor M (2002) Conformation of Glycomimetics in the free and proteinbound state: Structural and binding features of the C-glycosyl analogue of the core trisaccharide $\alpha$-D-Man- $(1 \rightarrow 3)$-[a-D-Man- $(1 \rightarrow 6)]-D$-Man. J Am Chem Soc 124: 14940-14951.

32. Yang GL, Franck RW, Bittman R, Samadder P, Arthur G (2001) Synthesis and growth inhibitory properties of Glucosamine-derived Glycerolipids. Org Lett 3 : 197-200.

33. Peng T, Wang L, Han FZ, Zhang SG, Wen XX (2009) The C-glycoside analogues of salidroside: synthesis and their protective effects on myocardial cells. J Med Chem 119: 89-96.

34. Zhang J, Wu ZG, Wang QW, Wang QF, Liu XY, et al. (2009) Design, synthesis of salidroside analogues and their antifatigue effect. J Fourth Mil Med Univ 30: 1916-1918.

35. Hwang SY, Shin JH, Hwang JS, Kim SY, Shin JA, et al. (2010) Glucosamine exerts a neuroprotective effect via suppression of inflammation in rat brain ischemia/reperfusion injury. Glia 58: 1881-1892.

36. Guo YB, Li XH, Zhao YH, Si YX, Zhu H, et al. (2011) Synthesis and biological evaluation of two salidroside analogues in the PC12 cell model exposed to hypoglycemia and serum limitation JChem Pharm Bull 59: 1045-1047.

37. Guo YB, Cheng Z, Wen X, Si YX, Dou SF, et al. (2013) Free radical scavenging and hepatoprotective effects of salidroside analogs on $\mathrm{CCl} 4$-induced cytotoxicity in LO2 cell J Med Chem Res 22: 2524-2530. 